\title{
Clinician advice, an interactive computer program, and motivational counselling increased smoking cessation in teens
}

Hollis JF, Polen MR, Whitlock EP, et al. Teen reach: outcomes from a randomized, controlled trial of a tobacco reduction program for teens seen in primary medical care. Pediatrics 2005;115:981-9.

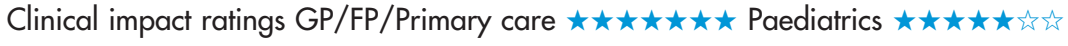

Does an intervention of brief clinician advice during routine medical visits, an interactive computer program, and brief motivational counselling reduce smoking in adolescent smokers and non-smokers over the long term?

\section{METHODS}

L

Design: randomised controlled trial (Teen Reach programme)

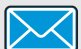

Allocation: $\left\{\right.$ concealed $\left.{ }^{*}\right\}$

Blinding: blinded \{data collectors and outcome assessors\}t.*

Follow up period: 1 and 2 years.

Setting: 7 large paediatric and family practice departments in group practice health maintenance organisation in Portland, Oregon and Vancouver, Washington, USA.

응 Participants: 2526 adolescents $14-17$ years of age $159 \%$ girls, $78 \%$ white) who were willing to stay after their clinician visit for about 15 minutes and had no intention to leave the geographic area in the next year.

$\mathrm{R}_{\mathbf{x}}$

Intervention: 1254 adolescents were allocated to the Teen Reach intervention, which comprised (1) a written prompt for primary care clinicians to encourage teens to quit smoking or to not start; (2) a 10-12 minute session on the Pathways to Change (PTC) interactive computer program that assessed the stage of readiness to start (or quit) smoking and then provided individualised advice; (3) 3-5 minutes of motivational counselling by trained health counsellors; and (4) 2 individual booster sessions with the PTC program and the health counsellor over the subsequent 11 months. 1272 adolescents were allocated to a die intervention (an attention control) and included 3-5 minutes of motivational counselling by health counsellors to promote increased consumption of fruit and vegetables.

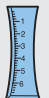

Outcomes: self reported smoking abstinence in the 30 days before 1 and 2 year assessments.

Patient follow up: $94 \%$ at 1 year and $88 \%$ at 2 years.

*See glossary.

tInformation provided by author.
MAIN RESULTS

The table shows the results.

\section{CONCLUSIONS}

A smoking cessation intervention comprising brief clinician advice during routine medical visits, an interactive computer program, and brief motivational counselling increased quitting in adolescent smokers at 2 years. The intervention reduced smoking onset in non-smokers at 1 year but not at 2 years

A modified version of this abstract appears in Evidence-Based Nursing.

\section{Commentary}

W know from systematic reviews that brief advice from a clinician and tailored self help interventions increase, by a modest amount, the number of adults who stop smoking compared with those who receive no intervention. ${ }^{12}$ The study of teenage smokers by Hollis et al provides encouraging evidence that brief interventions have similar effects in this age group. The study is distinctive in that it included interventions that encouraged abstinence in current smokers as well as prevention in current non-smokers.

Applying the results of the study by Hollis et al directly to clinical practice is not straightforward. Research staff delivered all aspects of the intervention other than the clinician's brief advice. As the authors acknowledge, most healthcare organisations are not set up to easily incorporate this package into routine clinical care. A cost effectiveness analysis would have provided helpful additional information for organisations considering whether to invest in such an intervention.

It is not possible to disaggregate the effects of clinician advice, motivational interviewing by a counsellor, and computerised self help in this study. Although the study does not directly support an effect of brief advice alone (and indeed only $41 \%$ of the intervention group recalled receiving clinician advice), we can be confident from other published evidence and systematic reviews that advice is worthwhile in encouraging smokers to stop. ${ }^{1}$ The study by Hollis et al provides less evidence that brief clinical interventions discourage non-smokers from starting. Based on current evidence, busy clinicians might justifiably prioritise their preventive efforts to focus on helping current smokers to quit. Tim Lancaster, MBBS University of Oxford Oxford, UK

For correspondence: Dr J $\mathrm{F}$ Hollis, Center for Healith Research, Kaise Permanente, Portland, OR, USA. jack.hollis@kpchr.org

Source of funding: National Cancer Institute.

Teen Reach smoking cessation intervention $v$ diet intervention (control) for smoking abstinence in adolescents*

\begin{tabular}{lllll}
\hline Baseline smoking status & Follow up & Event rates & RBI (95\% CI) & NNT (CI) \\
\hline All participants & 1 year & $77 \% v 73 \%$ & $6.1 \%(2$ to 10) & $23(14$ to 67$)$ \\
& 2 years & $73 \% v 69 \%$ & $6.2 \%(1$ to 11$)$ & $24(14$ to 158$)$ \\
Smokers (smoked in previous 30 d) & 1 year & $33 \% v 23 \%$ & $38 \%(4$ to 77$)$ & $12(6$ to 114$)$ \\
Non-smokers & 2 years & $30 \% v 21 \%$ & $39 \%(2$ to 84$)$ & $13(6$ to 304$)$ \\
& 1 year & $91 \% v 88 \%$ & $3.4 \%(0.1$ to 6) & $34(20$ to 949$)$ \\
\hline
\end{tabular}

*Abbreviations defined in glossary; RBI, NNT, and Cl calculated from control event rate and odds ratio (Cl) in article. Based on total $\mathrm{n}=2524$ with multiple imputation for missing values. 